Aim of the study: The authors present a novel and specific controlled ovarian stimulation protocol for fertility preservation in women with estrogen-positive receptor breast cancer undergoing neoadjuvant chemotherapy. The protocol foresees random start ovarian stimulation and the use of letrozole associated to tamoxifen.

Material and methods: Forty breast cancer patients were included in the study. COS was performed either with recombinant FSH or hMG. Concomitantly with $\operatorname{COS}$, letrozole in a dose of $5 \mathrm{mg}$ and tamoxifen in a dose of $20 \mathrm{mg}$ were given orally on a daily basis. The trigger was performed with $0.2 \mathrm{mg}$ of triptorelin, in the presence of follicles $\geq 19 \mathrm{~mm}$. Oocyte retrieval was scheduled 35-36 hours after triptorelin injection. Our main outcome measures were the number of oocytes collected and number of oocytes vitrified, the length of ovarian stimulation, total dose of gonadotropins administered, and levels of estradiol on the day of the trigger.

Results: The mean age of patients was $30.43 \pm 4.25$ years. Nineteen women commenced COS in the luteal phase, eleven in the early follicular phase and ten in the late follicular phase. The mean number of collected oocytes was $11.78 \pm 9.12$ and the mean number of vitrified oocytes was 9.72 \pm 7.36 . The mean duration of $\operatorname{COS}$ was $10.03 \pm 1.33$ days. The mean estradiol concentrations on the triggering day was $623.10 \pm 441.27$, and the mean dose of gonadotropins administered was $2540 \pm 713.10$.

Conclusions: The authors suggest that the protocol is efficient and may be a safe option for oocyte vitrification in these patients.

Key words: breast cancer, fertility preservation, ovarian stimulation, letrozole, tamoxifen.

Contemp Oncol (Pozn) 2017; 21 (4): 290-294 DOI: https://doi.org/10.5114/wo.2017.72395

\section{A specific controlled ovarian stimulation (COS) protocol for fertility preservation in women with breast cancer undergoing neoadjuvant chemotherapy}

Felipe Cavagna ${ }^{1,2}$, Anagloria Pontes ${ }^{2}$, Mario Cavagna ${ }^{1}$, Artur Dzik ${ }^{1}$, Nilka F. Donadio' ${ }^{1}$, Rafael Portela ${ }^{1}$, Michelle T. Nagai ${ }^{1}$, Luiz H. Gebrim ${ }^{1}$

'Women's Health Reference Center, Hospital Perola Byington, São Paulo, Brazil ${ }^{2}$ Department of Gynecology and Obstetrics, Botucatu Medical School, São Paulo State University - UNESP, Botucatu, São Paulo, Brazil

\section{Introduction}

Breast cancer is the most common malignancy in adult women, and in the United States, $5 \%-7 \%$ of cases of invasive breast cancer ( 11,000/year) occur in women who are under age 40 at diagnosis [1]. Given the advent of early breast cancer diagnosis and effective cancer treatments, survival rates following breast cancer are increasing, with a 5 -year survival rate over $80 \%$ $[2,3]$. This fact justifies the concern about chemotherapy-related gonadal toxicity in women with reproductive wishes. Chemotherapy treatment may have deleterious effects on the ovarian reserve, by affecting the resting pool of primordial follicles or the growing follicle population [3] Given that, it is important to consider early referral of breast cancer young patients to fertility specialists, in order to discuss fertility preservation procedures [4-6]. Among these, medical ovarian protection, ovarian tissue cryopreservation, oocytes or embryos cryopreservation are the most common fertility preservation strategies [7-9]. As occurs in conventional in vitro fertilization (IVF) techniques, to obtain oocytes or embryos for cryopreservation, controlled ovarian stimulation (COS) is the first step to be considered. Performing COS in breast cancer patients prior to surgery may promote concerns about the risks of delaying chemotherapy treatment and exposing a breast cancer patient to high estradiol levels consequent to multiple follicle development, mainly in hormone-receptor positive tumors [10]. To mitigate the effect of high estradiol levels, the use of aromatase inhibitors has been demonstrated to be safe and efficient [11, 12]. In this series of cases, we performed COS in estrogen-receptor positive breast cancer patients undergoing neoadjuvant chemotherapy, and we proposed the concomitant administration of letrozole and tamoxifen with gonadotropins, which may be a safe approach in such type of cancers. It is possible that their different mechanisms of action would be complementary, with the aromatase inhibitor decreasing the estrogen level and thus allowing tamoxifen to function more effectively as a competitive inhibitor with estradiol. In order to mitigate thromboembolic event, which is a serious complication of cancer [13] and which can be exacerbated by the use of tamoxifen [14], we proposed the prophylactic use of a low molecular weight heparin, enoxaparin, administered on a daily basis, throughout ovarian stimulation.

\section{Material and methods}

This is an observational cross sectional study of breast cancer patients undergoing COS for fertility preservation in a tertiary public hospital. Between 
November 2014 and December 2016, 40 women with hormone-receptor positive breast cancer and indication of neoadjuvant chemotherapy underwent random start COS for fertility preservation, in a public IVF center in Sao Paulo, Brazil. Given that we propose a specific COS protocol for women who have a breast cancer not removed, so that ovarian stimulation is performed in the presence of an estrogen receptor positive tumor, only patients receiving neoadjuvant chemotherapy were included. The patients were divided in three groups, according to the phase of the menstrual cycle:

Initial Follicular Phase Group (IFP, $n=11)$ : the COS was initiated in the beginning of the follicular phase, in which a dominant follicle $(>10 \mathrm{~mm})$ was not detected.

Late Follicular Phase Group (LFP, $n=19$ ): the COS was initiated in the late follicular phase, in the presence of a dominant follicle $>10 \mathrm{~mm}$;

Luteal Phase Group (LP, $n=10)$ : the COS was initiated in the luteal phase, with ecographic evidence of follicular rupture and (or) an endometrium of secretory pattern.

COS was performed either with recombinant FSH or hMG, in a daily dose of 150-300 IU. The gonadotropin starting dose was chosen according to the antral follicle count: 150 IU daily with $\geq 15$ antral follicles, 225 IU daily with $<15 \geq 10$ antral follicles and 300 IU daily with $<10$ antral follicles. When COS was initiated in the late follicular phase, with the presence of a follicle $>10 \mathrm{~mm}$, a GnRH antagonist was introduced concomitantly with the gonadotropin; otherwise, the antagonist was introduced in the presence of a follicle $\geq 13 \mathrm{~mm}$. Concomitantly with COS, letrozole in a dose of $5 \mathrm{mg}$ and tamoxifen in a dose of $20 \mathrm{mg}$ were given orally on a daily basis. Enoxaparin was given daily in a dose of $40 \mathrm{mg}$ subcutaneously, as a prophylactic measure. The trigger was performed with $0.2 \mathrm{mg}$ of triptorelin, in the presence of follicles $\geq 19 \mathrm{~mm}$. Oocyte retrieval was scheduled 35-36 hours after triptorelin injection. After the oocyte retrieval, the patients discontinued any medication. Our main outcome measures were the number of oocytes collected and number of oocytes vitrified, the length of ovarian stimulation, total dose of gonadotropins administered, and levels of estradiol on the day of the trigger. The secondary outcome measure was to determine whether there are differences in the outcomes according to the phase of the cycle in which COS was initiated.

\section{Criteria of inclusion and exclusion}

Patients diagnosed with hormone-positive breast cancer and indication of neoadjuvant chemotherapy were included in this investigation. Patients with advanced and metastatic disease and with age $>40$ years were not included in the fertility preservation program.

\section{Ethics}

This research was approved by the Committee of Ethics in Research of the Women's Health Reference Center, in Sao Paulo, on 29 October 2014, under the number 848.880 . All patients signed an informed consent for undergoing cOS with the specific protocol.

\section{Statistical analysis}

A hypothesis test was applied to evaluate the statistical differences between the groups. The Kruskal-Wallis test was used to compare the results between groups: IFP versus LFP, IFP vs LF and LFP vs LF. The level of statistical significance was considered to be a $p$-value of less than 0.05 .

\section{Results}

This study included 40 patients with hormone receptor positive breast cancer undergoing neoadjuvant chemotherapy. Among the 40 patients, 28 were classified as immunohistochemical subtype luminal $B$, and twelve as luminal HER2+. No patient's tumor was more advanced than stage IIIA. Neoadjuvant chemotherapy was performed with the aim of downstaging the tumor, allowing for conversion from mastectomy to conservative surgery. The chemotherapy regimen employed in patients undergoing neoadjuvant chemotherapy was cyclophosphamide and doxorubicin (four cycles) and taxol (four cycles). Patients with luminal HER2 tumors received adjuvant trastuzumab therapy. The mean age of patients was $30.43 \pm 4.25$ years (range 21-39). The average duration of COS was 10.03 \pm 1.33 days (range, $8-13$ ). In all 40 patients it was collected 459 oocytes. Of these, 399 (86.92\%) were in metaphase $1 \mathrm{l}$ and were vitrified. The remainders were in metaphase I or germinal vesicle stage, and were discarded. The mean number of collected oocytes was $11.78 \pm 9.12$ (range 1-38) and the mean number of vitrified oocytes was $9.72 \pm 7.36$ (range, 0-34). The mean total dose of FSH administered was $2540.00 \pm 713.10 \mathrm{IU}$, and the mean estradiol concentrations on the triggering day were $623.10 \pm 441.27 \mathrm{pg} / \mathrm{ml}$. Nineteen women commenced COS in the luteal phase (LP), eleven in the initial follicular phase (IFP) and ten in the late follicular phase (LFP). When comparing the outcomes according to the phase of the cycle in which COS was commenced, there were no significant differences in the number of oocytes collected and vitrified, ovarian stimulation length, total dose of gonadotropin administered and estradiol levels on the trigger day phase. The results are shown in Table 1. In Fig. 1, the box plots graphs show the overall mean values, standard deviation and outliers of the mean values.

\section{Discussion}

Currently, oocyte vitrification may be considered the gold standard in female onco- fertility preservation, although embryo cryopreservation is still performed for this purpose [15]. Regarding oocyte cryopreservation, it was shown that women treated of breast cancer who have their oocytes vitrified before chemotherapy have good IVF performance and good obstetric outcomes, when compared to age-matched patients [16]. To perform embryos or oocytes cryopreservation, COS is the first step to be considered. Regarding this issue, some concerns may emerge, such as delaying chemotherapy treatment and exposing a breast cancer patient to high estradiol levels, particularly when the tumor is present, as occurs in the cases of neoadjuvant chemotherapy. The stimulation required for oocyte retrieval could delay oncologic treatment, given that 
Table 1 . Outcomes in 40 breast cancer patients undergoing COS for fertility preservation prior to neoadjuvant chemotherapy

\begin{tabular}{|c|c|c|c|c|c|}
\hline Parameter & $\begin{array}{l}\text { All patients } \\
\quad(n=40)\end{array}$ & $\begin{array}{c}\text { IFP } \\
(n=11)\end{array}$ & $\begin{array}{c}\text { LFP } \\
(n=10)\end{array}$ & $\begin{array}{c}\text { LP } \\
(n=19)\end{array}$ & $P$-value \\
\hline Age (years) & $30.43 \pm 4.25$ & $30.73 \pm 3.52$ & $31 \pm 5.25$ & $29.95 \pm 4.24$ & 0.697 \\
\hline Collected oocytes & $11.78 \pm 9.12$ & $9.18 \pm 6.0$ & $10.90 \pm 10.77$ & $13.74 \pm 9.67$ & 0.173 \\
\hline Vitrified Oocytes & $9.72 \pm 7.36$ & $8.27 \pm 5.37$ & $7.90 \pm 6.44$ & $11.53 \pm 8.61$ & 0.331 \\
\hline Days of stimulation & $10.03 \pm 0.33$ & $10.00 \pm 1.41$ & $9.70 \pm 0.49$ & $10.21 \pm 1.23$ & 0.653 \\
\hline FSH/hMG total dose (IU) & $2,540 \pm 713.1$ & $2,677 \pm 544.3$ & $2,260 \pm 706.6$ & $2608 \pm 792.2$ & 0.983 \\
\hline Estradiol Levels (pg/ml) & $623.1 \pm 441.2$ & $783.0 \pm 411.7$ & $661.7 \pm 666.7$ & $510.2 \pm 274.8$ & 0.053 \\
\hline
\end{tabular}

Data are expressed as mean $\pm S D$.

IFP-initial follicular phase; LFP - late follicular phase; $L P$ - luteal phase

The limit of significance is a $p$ value $\leq 0.05$
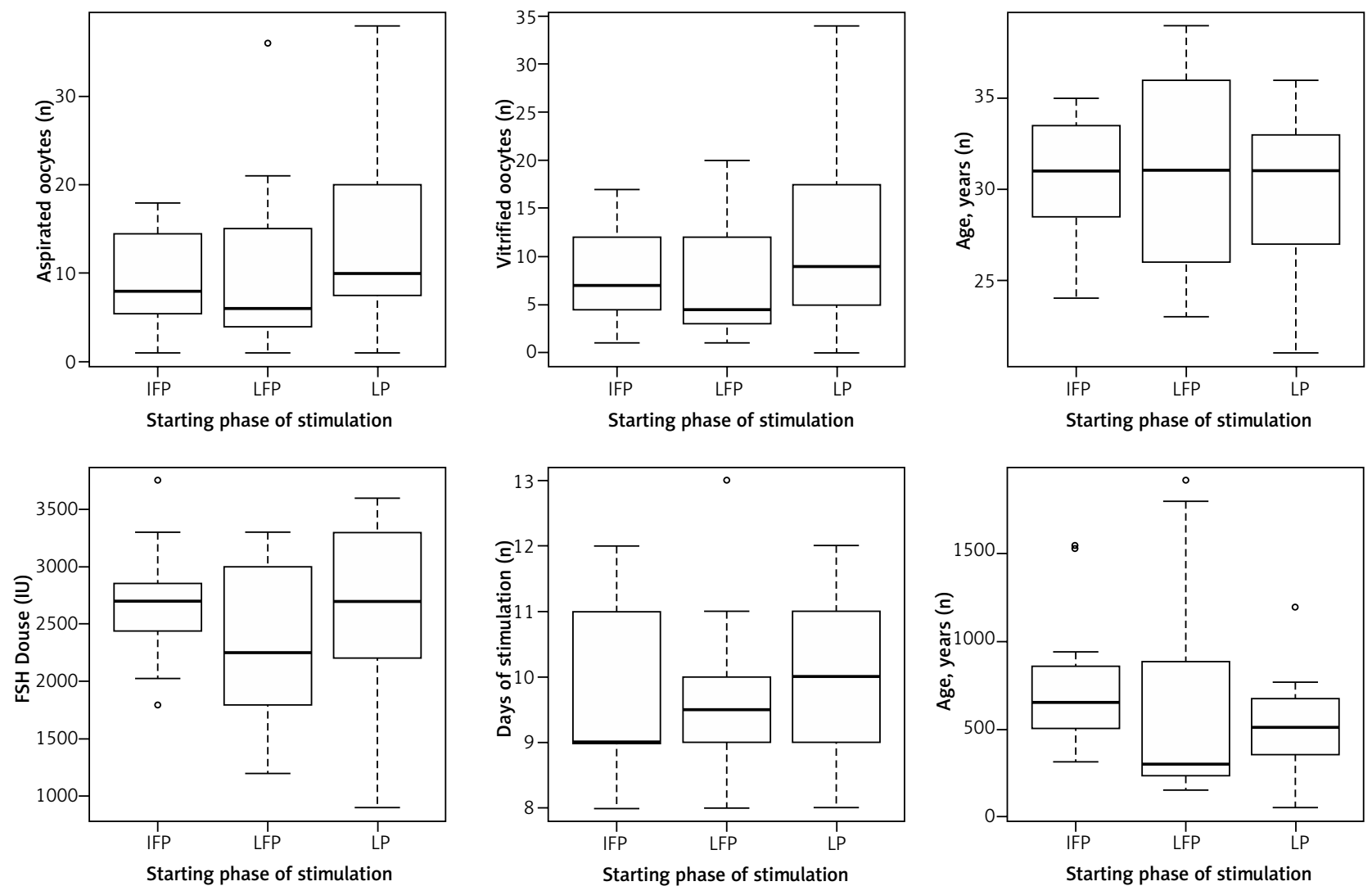

IFP - initial follicular phase; LFP - late follicular phase; $L P$ - luteal phase

Fig. 1. Box plot graph showing the results of random-start ovarian stimulation. The graph illustrates the median (inner black line), the upper and lower quartiles (the box) and outliers

the conventional COS, when initiated in the beginning of the follicular phase may require up to 6 weeks to be concluded. Random-start ovarian stimulation, which means initiating COS immediately, regardless of the patient's menstrual-cycle phase, has become a well-established approach in fertility preservation strategies, allowing oocyte retrieval in no more than two weeks, in the majority of the cases $[17,18]$. Currently, random-start ovarian stimulation is routinely and successfully employed for emergency IVF [19-21] and the outcome of ovarian stimulation seems to be similar after stimulation initiation during any phase of the menstrual cycles [22]. Recently, it was reported that the formation of euploid blastocyst does not depend on the phase of the cycle in which ovarian stimulation commences [23]. Another concern might be the high estradiol levels consequent to ovarian stimulation, particularly in hormone-positive breast cancer patients undergoing neoadjuvant chemotherapy. In order to reduce estradiol concentrations, ovarian stimulation with aromatase inhibitors has been proved to be an efficient procedure [11]. Recent 
publications also confirm that the adjuvant therapy with letrozole, throughout the length of the COS, is a safe and efficient approach [24-26]. The most employed aromatase inhibitor in ovarian stimulation protocols is letrozole, which has been proved to be more efficient than anastrozole for this purpose [27]. Another option to decrease estradiol levels could be the protocol performing profound LH suppression by high and sustained GnRH antagonist dosis, which maintains estradiol levels around the physiological range [28]. This approach, however, has the inconvenient of the high cost due to the elevated daily dose of the GnRH antagonist. When the indication is neodjuvant chemotherapy, and the cancer is hormone-receptor positive, the concerns about estradiol levels augment, because of the presence of the tumor during ovarian stimulation. In this study, we present a specific COS for patients undergoing neoadjuvant chemotherapy. Besides the use of letrozole, we also propose the administration of tamoxifen, which is a selective estrogen receptor modulator with antagonist effect on the breast. The use of letrozole together with tamoxifen may protect the patient by two different mechanisms of action: diminishing estradiol levels with the aromatase inhibitor and by competition in the estradiol receptors by tamoxifen. As well as letrozole, it was already demonstrated that tamoxifen can be safely employed to perform ovarian stimulation in breast cancer patients [29]. Meirow et al. [30] stated that co-administration of tamoxifen for fertility preservation does not interfere with ovarian stimulation outcomes and should be considered safe. The risk of thromboembolic complications can be minimized by the prophylactic use of a low molecular weight heparin, enoxaparin, in a daily basis. Being so, the random start COS prevents the delay to start chemotherapy, and the concomitant use of letrozole and tamoxifen may help to make the procedure safer. It is known that there is an association between tamoxifen therapy and thromboembolic events [31]. Furthermore, considering the possibility of venous thromboembolism as a paraneoplasic syndrome, we propose the prophylactic administration of enoxaparin in order to prevent this complication. The use of $\mathrm{GnRH}$ agonist to trigger final oocyte maturation avoids the occurrence of hyperstimulation ovarian syndrome, which is an important complication of ovarian stimulation, making the procedure safer [32]. There is no place for hCG trigger in COS for cryopreservation of oocytes or embryos. Our results showed an adequate number of oocytes retrieved and cryopreserved, with relative low concentrations of estradiol and without occurrence of any complications. The mean estradiol concentrations observed with this protocol $(623.10 \pm 44.27 \mathrm{pg} / \mathrm{ml})$ was considerably lower than in conventional COS for IVF, considering that serum estradiol levels during COS are increased by 10-fold compared with those of natural cycles [33]. Regarding the outcomes evaluated, it was not observed any statistically significant difference among the three groups studied (IFP, LFP and LP). In performing COS with this specific protocol, we observed a high rate of oocyte maturity (86.92\%) even considering that in fertility preservation procedures we generally aspirate all follicles, including those very small which would be ignored in a conventional IVF treatment. It was also ob- served that some patients, although young, have a poor response to ovarian stimulation. In a 34-year-old patient, we retrieved only one immature oocyte, and vitrification was not possible. It was previously registered that the number of oocytes retrieved in breast cancer patients with BRCA1 mutations is significantly lower compared to BRCA negative and untested patients [34]. On the other hand, it was recently described that women with gynecological cancer have less number of retrieved mature oocytes compared with haematological and breast cancer patients [35]. Interestingly, it was also recently reported that both healthy and cancer-affected BRCA mutation women have normal response to COS in IVF cycles [36]. However, the outcomes observed in this investigation were comparable to conventional IVF results, with significant lower estradiol levels. Obviously, women affected with BRCA mutations may have concerns about transmission of the mutation to offspring. In these cases, preimplantation genetic diagnosis to avoid the birth of affected offspring is ethically acceptable [37]. We postulate that the administration of tamoxifen, concomitantly with letrozole, could promote an additional safeness to the procedure, by competing with estrogen receptors. The results of this investigation suggest that a specific protocol of ovarian stimulation for fertility preservation in this group of patients may be an effective procedure, and no complications were observed during the treatment. Pharmacological ovarian stimulation before the initiation of chemotherapy seems to be safe, and one prospective study did not observe a negative impact on patient's survival after this procedure [25]. However, there remains a need for long-term follow up to better determine the safety of COS in patients with hormone-receptor positive breast cancer and indication of neoadjuvant chemotherapy, and we shall stress that our findings should be confirmed with more rigorous reporting and data monitoring in prospective trials of larger populations.

The authors declare no conflict of interest.

\section{References}

1. Kim SS, Klem J, Fabian C. Breast cancer and fertility preservation. Fertil Steril 2011; 95: 1535-43.

2. Siegel RL, Miller KD, Jemal A. Cancer Statistics, 2017. CA Cancer J Clin 2017; 67: 7-30.

3. Morgan S, Anderson RA, Gourley C, Wallace WH, Spears N. How do chemotherapeutic agents damage the ovary? Hum Reprod Update 2012; 18: 525-35.

4. Loren AW, Mangu PB, Beck LN, et al. Fertility preservation for patients with cancer: American Society of Clinical Oncology clinical practice guideline update. J Clin Oncol 2013; 31: 2500-10.

5. Peccatori FA, Azim HA Jr, Orecchia R, Hoekstra HJ, Pavlidis N, Kesic V, Pentheroudakis G; ESMO Guidelines Working Group. Cancer, pregnancy and fertility: ESMO Clinical Practice Guidelines for diagnosis, treatment and follow-up. Ann Oncol 2013; 24 (Suppl 6): vi160-70.

6. Paluch-Shimon S, Pagani O, Partridge AH, et al. Second international consensus guidelines for breast cancer in young women (BCY2). Breast 2016; 26: 87-99. 
7. Sonmezer M, Oktay K. Fertility preservation in young women undergoing breast cancer therapy. Oncologist 2006; 11: 422-34.

8. De Pedro M, Otero B, Martin B. Fertility preservation and breast cancer: a review. Ecancermedicalscience 2015; 9: 1-20.

9. Lambertini M, Cinquini M, Moschetti I, Peccatori FA, Anserini P, Valenzano-Menada M, Tomirotti M, Del Mastro L. Temporary ovarian suppression during chemotherapy to preserve ovarian function and fertility in breast cancer patients: A GRADE approach fo evidence evaluation and recommendations by the Italian Association of Medical Oncology. Eur J Cancer 2017; 71: 25-33.

10. Azim AA, Costantini-Ferrando M, Oktay K. Safety of fertility preservation by ovarian stimulation with letrozole and gonadotropins in patients with breast cancer: a prospective controlled study. J Clin Oncol 2008; 26: 2630-5.

11. Reddy J, Oktay K. Ovarian stimulation and fertility preservation with the use of aromatase inhibitors in women with breast cancer. Fertil Steril 2012; 98: 1363-9.

12. Checa-Vizcaíno MA, Corchado AR, Cuadri ME, Comadran MG, Brassesco M, Carreras R. The effects of letrozole on ovarian stimu lation for fertility preservation in cancer-affected women. Reprod Biomed Online 2012; 24: 606-10.

13. Brand JS, Hedayati E, Bhoo-Pathy N, Bergh J, Hall P, Humphreys K, Ludvigsson JF, Czene K. Time-dependent risk and predictors of venous thromboembolism in breast cancer patients: A population-based cohort study. Cancer 2016; 123: 468-75

14. Anderson JA, Weitz Jl. Hypercoagulable states. Clin Chest Med 2010; 31: 659-73.

15. Rienzi L, Ubaldi FM. Oocyte versus embryo cryopreservation for fertility preservation in cancer patients: guaranteeing a women's autonomy. J Assist Reprod Genet 2015; 32: 1195-6.

16. Martinez M, Rabadan S, Domingo J, Cobo A, Pellicer A, Garcia-Velasco JA. Obstetric outcome after oocyte vitrification and warming for fertility preservation in women with cancer. Reprod. Biomed. Online 2014; 29: 722-8.

17. Cakmak H, Rosen MP. Random-start ovarian stimulation in patients with cancer. Curr Opin Obstet Gynecol 2015; 27: 215-21.

18. Mangili G, Papaleo E, Sigismondi C, et al. Timing should no longer be an obstacle to oocyte cryopreservation in patients with cancer. Tumori 2016; doi: 10.5301/tj.5000586.

19. Rashidi BH, Tehrani ES, Ghaffari F. Ovarian stimulation for emergency fertility preservation in cancer patients: A case series study. Gynecol Oncol Rep 2014; 10: 19-21.

20. Kim JH, Kim SK, Lee HJ, Lee JR, Jee BC, Suh CS, Kim SH. Efficacy of random-start controlled ovarian stimulation in cancer patients. J Korean Med Sci 2015; 30: 290-5.

21. Robertson DM, Gilchrist RB, Ledger WL, Baerwald A. Random start or emergency IVF/in vitro maturation: a new rapid approach to fertility preservation. Womens Health (Lond) 2016; 12: 339-49.

22. von Wolff M, Capp E, Jauckus J, Strowitzki T, Germeyer A; FertiPROTEKT study group. Timing of ovarian stimulation in patients prior to gonadotoxic therapy: an analysis of 684 stimulations. Eur J Obstet Gynecol Reprod Biol 2016; 199: 146-9.

23. Ubaldi FM, Capalbo A, Vaiarelli A, et al. Follicular versus luteal phase ovarian stimulation during the same menstrual cycle (DuoStim) in a reduced ovarian reserve population results in a similar euploid blastocyst formation rate: new insight in ovarian reserve exploitation. Fertil Steril 2016; 105: 1488-95.

24. Domingo J, Garcia-Velasco JA. Oocyte cryopreservation for fertility preservation in women with cancer. Curr Opin Endocrinol Diabetes Obes 2016; 23: 465-9.

25. Kim J, Turan V, Oktay K. Long-term safety of letrozole and gonadotropin stimulation for fertility preservation in women with breast cancer. J Clin Endocrinol Metab 2016; 101: 1364-71.

26. Pereira N, Hancock K, Cordeiro CN, Lekovich JP, Schattman GL, Rosenwaks Z. Comparison of ovarian stimulation response in patients with breast cancer undergoing ovarian stimulation with letrozole and gonadotropins to patients undergoing ovarian stimulation with gonadotropins alone for elective cryopreservation of oocytes. Gynecol Endocrinol 2016; 32: 823-6

27. Azim AA, Costantini-Ferrando M, Lostritto K, Oktay K. Relative Potencies of Anastrozole and Letrozole to Suppress Estradiol in
Breast Cancer Patients Undergoing Ovarian Stimulation before in Vitro Fertilization. J Clin Endocrinol Metab 2007; 92: 2197-200.

28. Adda-Herzog E, Gallot V, Le Bras A, Hesters L, Fanchin R. Natura ovarian stimulation (NATOS): an innovative estradiol-sparing, multiple follicle protocol suitable for fertility preservation in women with breast cancer. Hum Reprod 2014; 29 (suppl 1): i241.

29. Oktay K, Buyuk E, Davis O, Yermakova I, Veeck L, Rosenwaks Z. Fertility preservation in breast cancer patients: IVF and embryo cryopreservation after ovarian stimulation with tamoxifen. Hum Reprod 2003; 18: 90-5.

30. Meirow D, Raanani H, Maman E, et al. Tamoxifen co-administration during controlled ovarian hyperstimulation for in vitro fertilization in breast cancer patients increases the safety of fertility-preservation treatment strategies. Fertil Steril 2014; 102: 488-95.

31. Parikh RP, Odom EB, Yu L, Colditz GA, Myckatyn TM. Complications and thromboembolic events associated with tamoxifen therapy in patients with breast cancer undergoing microvascular breast reconstruction: a systematic review and meta-analysis. Breast Cancer Res Treat 2017; 163: 1-10.

32. Thomsen L, Humaidan P. Ovarian hyperstimulation syndrome in the 21st century: the role of gonadotropin-releasing hormone ag onist trigger and kisspeptin. Curr Opin Obstet Gynecol 2015; 27 : 210-4

33. Joo BS, Park SH, An BM, Kim KS, Moon SE, Moon HS, Serum estradiol levels during controlled ovarian hyperstimulation influence the pregnancy outcome of in vitro fertilization in a concentration-dependent manner. Fertil Steril 2010; 93: 442-6.

34. Oktay K, Kim JY, Barad D, Babayev SN. Association of BRCA1 mutations with occult primary ovarian insufficiency: a possible explanation for the link between infertility and breast/ovarian cancer risks. J Clin Oncol 2010; 28: 240-4

35. Alvarez M, Ramanathan P. Fertility preservation in female oncology patients: the influence of the type of cancer on ovarian stimulation response. Hum Reprod 2016; doi: 10.1093/humrep/dew158.

36. Shapira M, Raanani H, Feldman B, et al. BRCA mutation carriers show normal ovarian response in in vitro fertilization cycles. Fertil Steril 2015; 104: 1162-7.

37. The Ethics Committee of the American Society for Reproductive Medicine. Fertility preservation and reproduction in cancer patients. Fertil Steril 2005; 83: 1622-8.

\section{Address for correspondence}

\section{Felipe Cavagna}

Women's Health Reference Center

Av. Brigadeiro Luis Antonio, 683

01317-001 Sao Paulo, Brazil

e-mail: cavagna@hotmail.com

Submitted: 29.042017

Accepted: $\quad 26.08 .2017$ 